\title{
Manipulating Deformable Linear Objects: Robot Motions in Single and Multiple Contact Points
}

\author{
Thorsten W. SCHMIDT and Dominik HENRICH \\ Embedded Systems and Robotics Lab. (RESY) \\ Faculty of Informatics, Building 48 \\ University of Kaiserslautern, D-67653 Kaiserslautern, Germany \\ E-Mail: tws@web.de, henrich@informatik.uni-kl.de, \\ Http: //resy.informatik.uni-kl.de/
}

\begin{abstract}
The task of handling non-rigid one-dimensional objects by a robot manipulation system is investigated. Especially, approaches to calculate motions with specific behavior in point contacts between the object and environment are regarded. For single point contacts, motions based on generalized rotations solving the direct and inverse manipulation problem are investigated. The latter problem is additionally tackled by simple rotation and translation motions. For double and multiple point contacts, motions based on Splines are suggested. In experimental results with steel springs, the predicted and measured effect for each approach are compared.
\end{abstract}

\section{INTRODUCTION}

The manipulation of rigid objects by robots has been investigated for several decades, but relatively little work regarding the manipulation of non-rigid or deformable objects has been performed. A recent overview is provided in [Henrich00a]. Here, we focus on deformable onedimensional linear objects (DLOs), such as cables, wires, ropes, strings, beams, etc. This task has various application fields, for example, hot-wire maintenance, cable form assembly, or production of control cabinets. The main problem of manipulating these objects is that they may change their shape during manipulation.

To cope with this problem, one approach is to estimate the shape of the deformable objects by calculating an internal model and simulate the object behavior. A static model for objects and obstacles can be calculated in two [Hirai94] or three [Wakamatsu95] dimensions. An extension leads to a dynamic model of deformable linear objects [Wakamatsu97]. Plastic deformations and a faster approach for treating workpiece dynamics are given in [Remde99c]. However, it is not clear how to use the object models to control the robot motion. For more complex objects (inhomogeneous or non-isotrop), the characteristic material parameters are difficult to determine. Finally, the shape calculation can be very time consuming.

Here, we take a simple analytical open-loop approach to predict the object's shape approximately. The approach is based on the formulation of contact states of the DLO in an environment of rigid convex objects [Henrich99a]. Here, we concentrate on edge/edge point contacts, since they occur in many assembly tasks, such as inserting a
DLO into a tube, hooking a DLO over a beam, or establishing a new contact without losing the current ones. Additionally, the edge/edge point contacts are stable, thus, small movements will not lead to a change of the contact state.

All robot motions are calculated with a minimal knowledge of the environment. This knowledge is provided by two characteristic points at the DLO. The contact point is the location at the DLO, where the DLO is in contact with an obstacle. The effect point, is the location at the DLO, where the robot grips the DLO. Additionally, the DLO's tangent and normal vector in these points are regarded defining local coordinate systems. (In contact points, the normal vector is pointing away from the obstacle.) From the environment, we need only to know the contact points, the tangents of the DLO at these points, and the position and orientation of the robot in the effect point.

When manipulating deformable objects (especially DLOs), there are two different kind of problems. The first problem is the direct manipulation problem, where a robot motion in the effect point is specified by the user and the resulting motions of the DLO in the contact points are searched. The second is the inverse manipulation problem, where a motion of the DLO at the contact point is specified and the necessary motion of the robot in the effect point is searched. Note, that these problems exist only for deformable objects. In contrast to the behaviour of rigid objects, with deformable objects, the motions of robot and object are not necessarily identical, since the object may change its shape. Whenever deformable objects need to be handled in real applications, either the direct and/or the inverse manipulation problem need to be solved.

To cut down the complexity of these two problems, we distinguish qualitatively different DLO motion types at the contact point [Henrich99a]. The motions are defined in a projection of the objects onto a 2-dimensional plane orthogonal to the obstacle's edge (see Fig. 1). The rotate or slide motion does not change the contact state but changes the orientation or position of the DLO respectively. Additionally, the slide motion is subdivided into left slide $\left(S_{-}\right)$, right slide $\left(S_{+}\right)$and no slide $\left(S_{0}\right)$. The rotation motion is subdivided into negative or clock-wise rotation $\left(R_{-}\right)$, positive or counter-clockwise rotation $\left(R_{+}\right)$, 
and no rotation $\left(R_{0}\right)$. Note, that the slide and rotation motions are independently and that all possible DLO motions at a contact point can be composed with these two motion types.

Additionally, we assume, that the force necessary to deform the DLO is greater than the gravity force (DLOs in class E- of [Henrich99a]).

In this paper, we use the basic motion types to solve the direct and inverse manipulation problem of DLOs in point contacts. To do this, we have to come up with an answer to the following questions: How can DLOs with a single contact point be manipulated (Section 2)? What algorithms solve the direct (Section 2.1) and inverse (Section 2.2 and 2.3) motion problem? How can the solution to the inverse problem be extended to double contacts (Section 3)? What is the general formulation for multiple contact points (Section 4)?

\section{SINGLE POINT CONTACTS}

In this section, we first give an algorithm to solve the direct manipulation problem (CP-Move) and show two experimental results. Second, we give two algorithms to solve the inverse manipulation problem (CP-Move and RT-Move) and perform one experiment to show the possible movements.

\subsection{Direct manipulation problem}

For the first approach we assume the set-up of Fig. 1. The contact point $c$ of the DLO with the obstacle and the effect point $e$ are given. Also the tangent $T$ of the DLO in $c$ is known. The DLO may be bent, but the straight line from $c$ to $e$ has to be more or less parallel with the tangent $T$.

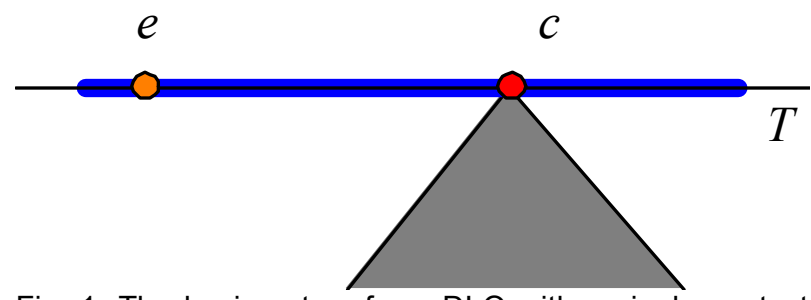

Fig. 1: The basic set-up for a DLO with a single contact point $c$ with the obstacle and the tangent $T$ in $c$. The effect point $e$ (including position and orientation) is moved by the robot.

In general, an arbitrary object can be moved in space by translations, rotations, or any combination of these movements. Rotations can be characterized by the rotation centre and rotation angle. Translations can be viewed as a special case of generalized rotations with the rotation centres lying in infinity perpendicular to the direction of the translation. Thus, it is sufficient to regard only (generalized) rotations of the effect point $e$ and translations can be omitted.

To solve the direct manipulation problem, we are interested in the resulting DLO motion in $c$ (in qualitative terms of rotation $R$ and slide $S$ ), for a given generalized rotation of the effect point $e$. To predict the resulting DLO motion, we perform a more general version of the experiments in [Henrich99a] by taking curved DLOs. Therefore, a $1 \mathrm{~cm} \times 1 \mathrm{~cm}$ grid is placed into the working area with a DLO in single contact with an obstacle. Every grid point represents a possible rotation centre for $e$. For every rotation centre, the resulting qualitative DLO motion ( $R$ and $S$ ) is observed in $c$. The observed DLO motions form clusters (areas) of rotation centres with identical DLO motions. For one specific set-up these areas are shown in Fig. 2. Note, that especially the borders of areas have been explored in the figure.

We have performed several experiments of this type while varying different parameters of the set-up. The varied parameters include the DLO material, the distance between $c$ and $e$, and the curvature of the DLO. All these experiments result in similar rotation centre areas. The boarders of these areas can be approximated in a general way by three characteristic lines of the set-up. These lines are the DLO normal $N$, the line $S_{0}$ through $e$ and $c$, and a line $R_{0}$ perpendicular to $S_{0}$ intersecting $S_{0}$ between $e$ and $c$. Although, we could not quantify the exact intersection point of $R_{0}$ and $S_{0}$ for all DLO materials in a general rule, the proportion between $e$ and $c$ stays the same for one DLO. Thus, for a particular DLO, the area boarders are approximated quite well by $N, S_{0}, R_{0}$ even when other setup parameters are varied.

Now, the algorithm CP-Move can be formulated as follows. For a particular DLO, determine the (constant) proportion of the intersection point. Then, for a given setup, calculate the location of the lines $S_{0}, R_{0}$ using $e, c$, and $N$. For any given robot motion in $e$, calculate the corresponding general rotation centre. Calculate the DLO motion area in which the rotation centre is located. Output the predicted qualitative DLO motion, which is determined by the specific area of the rotation centre.

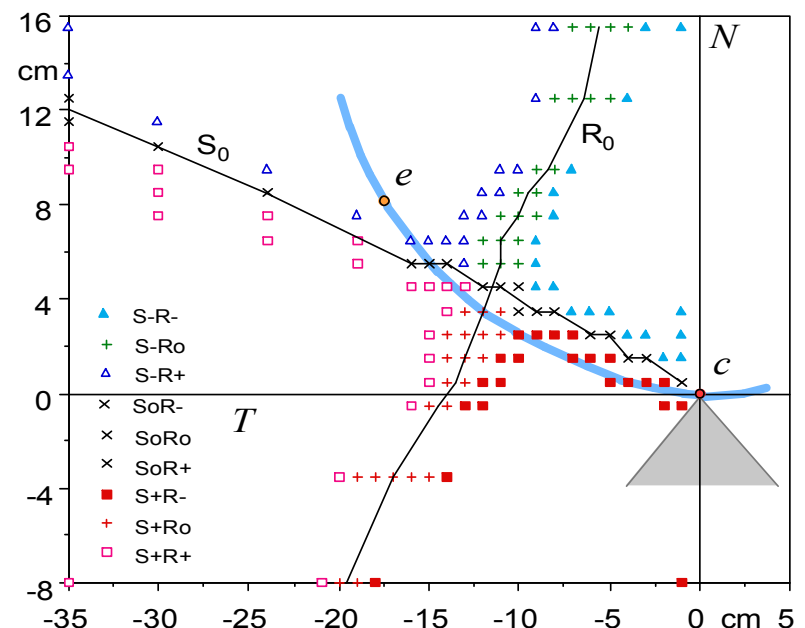

Fig. 2: Different rotation centres and the resulting motion in contact point $c$ for a clockwise rotation of effect point $e$ lying to the left of $c$ in $20 \mathrm{~cm}$ distance for a curved DLO. 


\subsection{Inverse manipulation problem solved by CP-Move}

To solve the inverse manipulation problem, we can use the DLO motion areas provided by $N, S_{0}, R_{0}$ (Section 2.1) and determine for a desired DLO motion a rotation centre in the corresponding area. The goal is to find those centres that have the greatest effect in $c$. A good rotation centre for one particular DLO motion is in most cases the one that has the largest average distance to the other areas. Experimental results show that for the motion type $S_{+} R_{-}$ or $S \_R_{+}$, the best rotation centre is not located in the infinite. Furthermore, there is a motion $R_{0} S_{0}$ which has only one rotation centre; the intersection point of the $R_{0}$ and $S_{0}$ line. The motion $R_{0} S_{0}$ implies that there is no DLO motion in $c$, but the DLO is deformed so that it does not make a rotation or slide.

Problems will occur if one of the set-up assumptions no longer holds true. For example, if a rotation of the DLO results in a deformation, then the straight line $S_{0}$ is sloping too (see Fig. 2). This changes the boarders of the areas and the rotation centre may move in a different area. So, the resulting effect is different from the desired one. Another problem is the rotation of the DLO at the contact point $c$. With its rotation, the rotation centre may also move to another area. As a result, CP-Move is only able to perform small movements correctly.

Additionally, it is not possible to quantify the amount of the movement. A rotation in the contact point $c$ can be reversed (for example, $R_{+}$by $R_{-}$), but the DLO deforms more and more if these two movements are repeated several times. After a number of iterations, the starting point cannot be reached again.

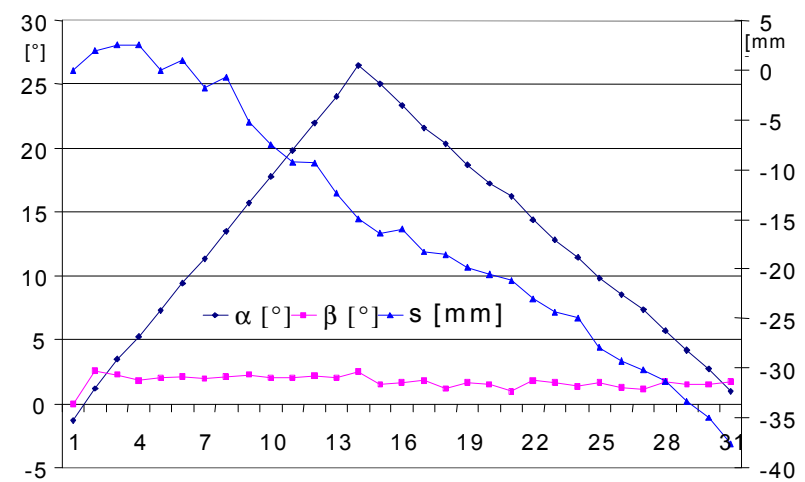

Fig. 3: Experimental results for CP-Move with a single contact point $c$, performing first a positive rotation $\left(R_{+}\right)$, then a negative rotation $\left(R_{-}\right)$of the DLO. Angle $\alpha$ is the rotation of the DLO in the contact point $c$ after step $i$. Distance $s$ is the undesired slide of the DLO in $c$. The value of angle $\beta$ indicates the rotation for each step.

To demonstrate the properties of CP-Move, we use a steel ruler as DLO (as in all of our experiments). This ruler has the advantage of being independent to gravity and it meets our general assumption of being unbent if no force is influencing it. Furthermore, only the 2D projection of the environment is regarded. First, we demonstrate a positive rotation $\left(R_{+}\right)$of about $25^{\circ}$ followed by a negative rotation of about the same amount (see Fig. 3) and second, a negative rotation $\left(R_{-}\right)$of circa $25^{\circ}$ and the same amount backwards (see Fig. 4). The set-up of the experiment is the same as shown in Fig. 1. The first experiment shows that the amount of rotation resulting from every step is different for positive and negative rotations. Thus, the way back requires 17 steps instead of 14 . Also, there is a unwanted slide of the DLO at the contact point. Related to the steps needed to obtain the desired angle, the second experiment is symmetric to the first one - the positive rotation is stronger than the negative rotation. There are 19 steps required for the first part and 17 steps for the second part to reach the starting rotation angle of the DLO at the contact point $c$. However, the total of the undesired translation increases slowly in the first part and then much faster in the second part of the movement.

As a result, this algorithm gives us the ability to handle curved DLOs. Because of the counter clockwise rotations of the effect point, the movements cannot made undone and the curvature of the DLO will increase till no more movements are possible.

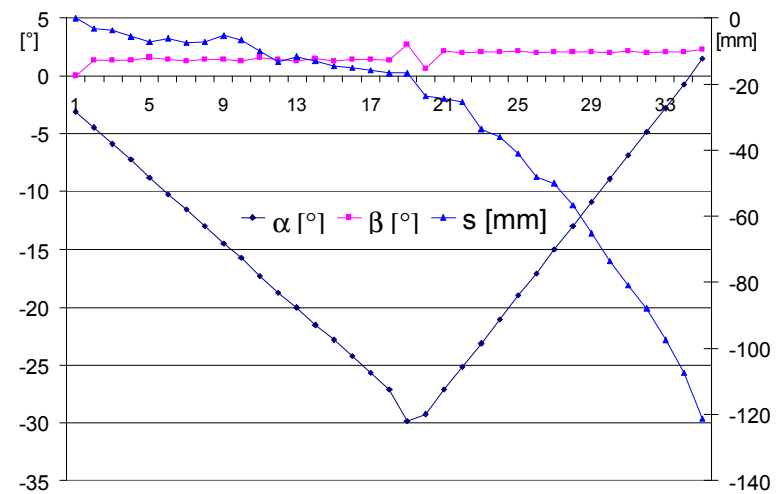

Fig. 4: Experimental set-up as in Fig. 3, but with first a negative rotation $\left(R_{-}\right)$, then a positive rotation $\left(R_{+}\right)$of the DLO.

\subsection{Inverse manipulation problem solved by RT-Move}

Due to the above problems, we come up with another algorithm, for solving the inverse manipulation problem, called RT-Move. We make almost the same assumptions as we did with CP-Move. As in Fig. 1, the tangent $T$ must be known. Additionally, the contact point $c$ of the DLO with the obstacle and the effect point $e$ must be given. The DLO may be bent. To best perform the movements, there should be a small contact pressure at the contact point $c$.

RT-Move is an approach to solve the inverse manipulation problem for DLOs at point contact. The desired rotation and the slide at the contact point $c$ are given and with that information, the necessary robot motion of the effect point $e$ is calculated. To perform a translation in positive direction $\left(S_{+}\right)$, the effect point $e$ is moved in the direction of the tangent $T$ (see Fig. 5). It is possible to specify the amount of the translation and with that translation there is 
no rotation at the contact point $c$. Additionally, we need to calculate the rotation in such a manner that it does not include any translation portion. This requirement is met by rotating the effect point $e$ around $c$ (see Fig. 5). While moving along that circle, the distance between $c$ and $e$ remains unchanged and therefore there is no translation. Because those two movements, rotation and translation, are independent, it is possible to combine them into one single movement.

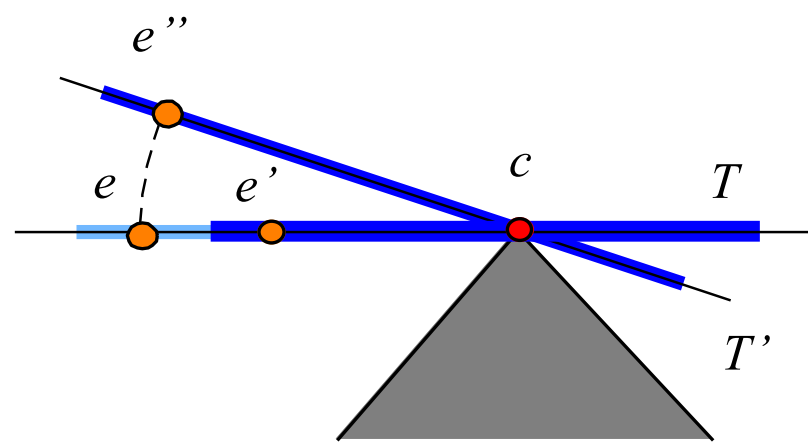

Fig. 5: DLO with tangent $T$ in contact point $c$. The effect point $e$ is shifted to $e$ ' or rotated around $c$ to $e$ ".

With RT-Move it is possible to specify the exact amount of translation and rotation the DLO should perform in the contact point $c$. The degree of the deformation will not change within the motions. Additionally, all movements have their inverse counterpart. For example, a positive rotation can be reversed with a negative rotation of the same amount.

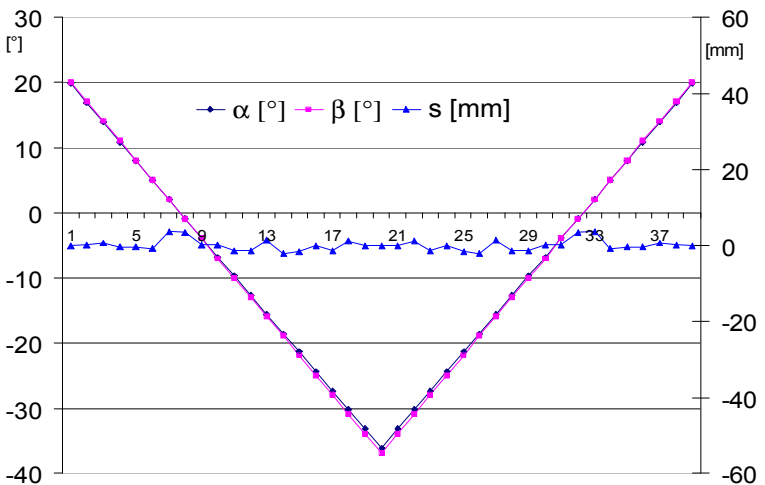

Fig. 6: Experimental results for RT-Move with a single contact point $c$, performing first a negative rotation $\left(R_{-}\right)$, then a positive rotation $\left(R_{+}\right)$of the DLO. The angle $\alpha$ is the desired rotation of the DLO in the contact point $c$ after step $i$, and the angle $\beta$ indicates the amount of rotation after every step. The distance $s$ is the undesired slide of the DLO in $c$.

The set-up of the experiment and the experiment itself differ slightly from those used for CP-Move (see Fig. 6). At the starting position, the DLO has already been rotated by $20^{\circ}$. Then there are 19 single negative rotations $\left(R_{-}\right)$of $3^{\circ}$ each. After those 19 steps the way back will be made with 19 positive rotations $\left(R_{+}\right)$, again of $3^{\circ}$ each. After those movements have been performed, the effect point $e$ is the same again $( \pm 0.3 \mathrm{~mm})$. The measured undesired slide is very small and will fluctuate within the accuracy of the measurement.

Thus, for the inverse manipulation problem in point contacts, RT-Move has the same capability as CP-Move, but it is able to undo the movements without changing the curvature of the DLO. Unfortunately, RT-Move does not solve the direct manipulation problem, since the robot motions dependent on the contact point $c$.

\section{DOUBLE POINT CONTACTS}

To manipulate DLOs with two contact points, we introduce an approach based on Splines, called S-Move. For this, the two contact points $c_{1}$ and $c_{2}$ and the tangents $T_{1}$ and $T_{2}$ of the DLO at these contact points are assumed to be known (see Fig. 7). The effect point $e$ is located somewhere between $c_{1}$ and $c_{2}$. If this is not the case, the parts of the DLO outside $c_{1}$ and $c_{2}$ are approximated the linear extrapolation of the corresponding tangent vector in $c_{1}$ and $c_{2}$, respectively.

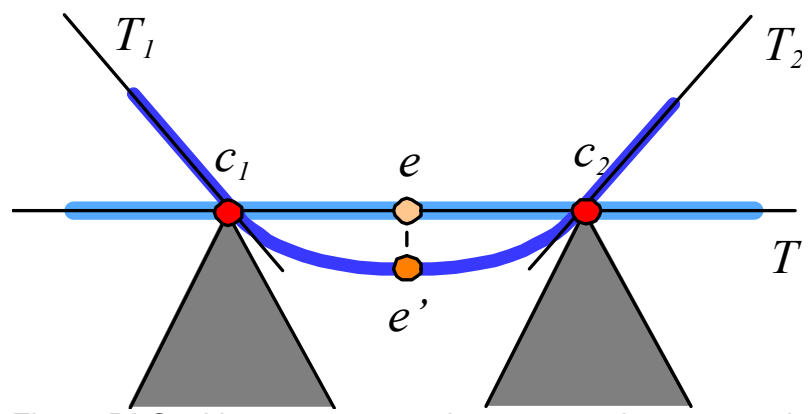

Fig. 7: DLO with two contact points $c_{1}, c_{2}$ and corresponding tangents $T_{1}, T_{2}$ after the effect point $e$ has been moved $e^{\prime}$.

To describe S-Move with two point contacts, we introduce the normal vector $N_{\mathrm{i}}$ for the two contact points $c_{i}, i=$ 1,2. The normal $N_{i}=T_{i} \times E_{i}$ is orthogonal to the DLO tangent $T_{i}$ at $c_{i}$ and to the contact edge $E_{i}$. Additionally, we assume that the vector $N_{i}$ is directed away from the obstacle's surface. If we regard $N_{i}$, there are only two different interesting cases for the general set-up of two contact points: (1) $N_{1}$ in the same direction then $N_{2}$ (Fig. 7) and (2) $N_{1}$ in the opposite direction then $N_{2}$ (Fig. 8). All other angles between $N_{1}$ and $N_{2}$ are similar to one of these two cases. In Case 1 , the possible movement of $e$ that changes the tangents $T_{1}$ and $T_{2}$ but does not lose a contact point is a movement of $e$ in the direction opposite to $N_{1}$ and $N_{2}$. In Case 2, a possible movement of $e$ that rotates $T_{1}$ and $T_{2}$ of the DLO at $c_{1}$ and $c_{2}$ without losing a contact point is the rotation of $e$ around itself.

If we regard all possible combinations of DLO motion at $c_{i}$, we recognize that it is impossible to make a rotation or slide at one contact point without causing an (undesired) motion at the other contact point. Because of this, we are unable to calculate one motion of $e$ that results exactly in the desired motion at both contact points. To 
solve this problem, our approach is to approximate the DLO with a Spline of third order and to treat rotation and slide in $c_{i}$ separately.

To obtain the desired rotation in $c_{i}$, we calculate the Spline based on its four variables. These variables determined by the two contact points and the tangents at the two contact points. The tangents $T_{1}$ and $T_{2}$ are selected according to the desired DLO rotation at $c_{1}$ and $c_{2}$. By calculating the shape of the Spline, we can determine the new effect point $e$ '. Based on the positions and Spline tangents of $e$ and $e^{\prime}$, we can determine the necessary translation and rotation the robot has to perform to obtain the desired DLO rotation at $c_{1}$ and $c_{2}$.

To obtain the desired slides in $c_{i}$, a translation of $e$ is calculated by the vector sum of all slide vectors $t_{i}$. (The $t_{i}$ are pointing in the direction of $T_{i}$ and having the length of the desired slide at $c_{i}$.) The length of the resulting vector is divided by the number of contact points (here two). The resulting vector is the best possible translation of $e$ to perform the desired slides at $c_{i}$.

It is possible to calculate whether the specified motion will keep or lose one or more contact points. The normal vector of the Spline at a contact point should point away from the obstacle (same direction as $N_{i}$ ). The direction of the normal vector is calculated by the second derivative. If the second derivative is positive, the Spline is left bent at the contact point. Therefore, the tangent $T$ must be rotated counter clockwise. If it is negative, the tangent must be rotated clockwise.

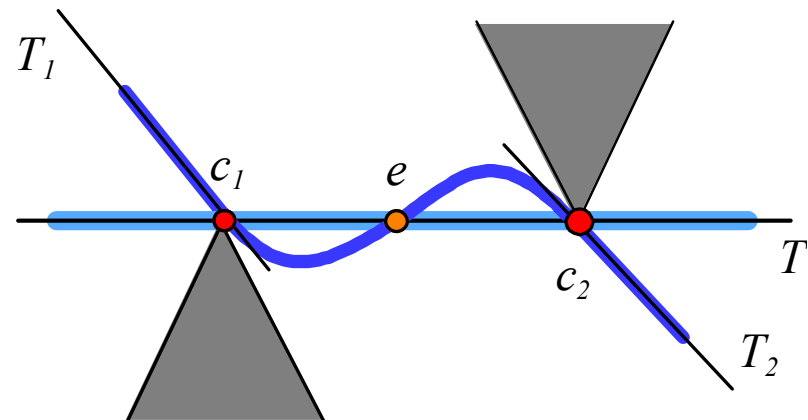

Fig. 8: DLO with two contact points $c_{1}, c_{2}$ and corresponding tangents $T_{1}, T_{2}$ after the effect point $e$ has been rotated around itself.

$\mathrm{S}-$ Move is able to perform the given motions in given quantified amounts. It is possible to specify the exact amount of translation and rotation the DLO should undergo at the contact points $c_{1}$ and $c_{2}$. It is most likely that the DLO is deformed during the motions. An advantage is that all movements can be reversed. For example, a positive rotation at a certain contact point can be reversed with a negative rotation of the equal amount at the same contact point. A disadvantage is that not all motions are performed in exactly the desired manner (see Fig. 7). For example even if a rotation at $c_{1}$ and none at $c_{2}$ is requested, there will be also a rotation to $c_{2}$.

For each of the above cases, we show the corresponding experiments, with one set-up like Fig. 7 (Case 1) and one such as in Fig. 8 (Case 2).

In the first experiment, the motion of the DLO is at the contact point $c_{1}: R_{-}$and at $c_{2}: R_{+}$. At both contact points, the given amount of the rotation is $3^{\circ}$ for every step. After rotating $27^{\circ}$ ( 9 steps), the inverse motion (at $c_{1}: R_{+}$and at $\left.c_{2}: R_{-}\right)$is carried out. Fig. 9 shows the results of the movement. The motion is in every step a few degrees smaller, because of the lag after the first step. Because the motions at two contact points are reversible, the graph is symmetric. The translation in- and decreases steadily.

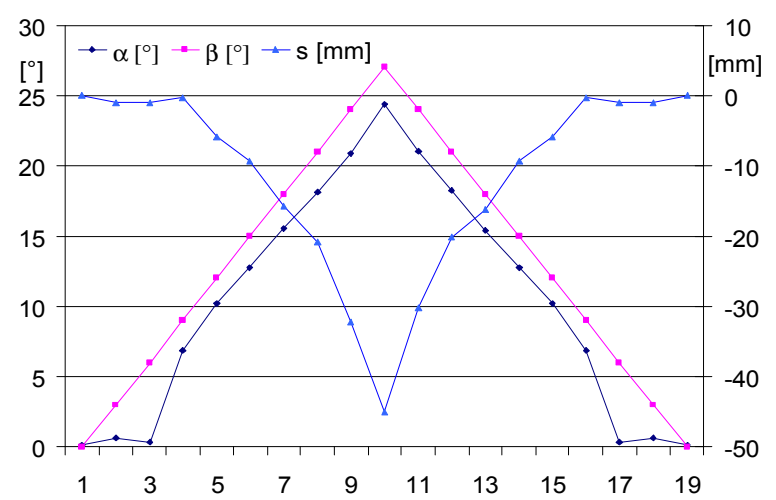

Fig. 9: Experimental results for S-Move for a negative rotation $\left(R_{-}\right)$at contact point $c_{1}$ and a positive rotation $\left(R_{+}\right)$ at $c_{2}$. The angle $\alpha$ is the desired rotation of the DLO at the contact point $c$ after step $i$, and the angle $\beta$ indicates the amount of rotation after every step. The distance $s\left(s_{1}=s_{2}\right)$ indicates the undesired slide of the DLO at $c_{1}$ and $c_{2}$. Here, $s_{1}=s_{2}$ hold true, since the experiment is symmetrical.

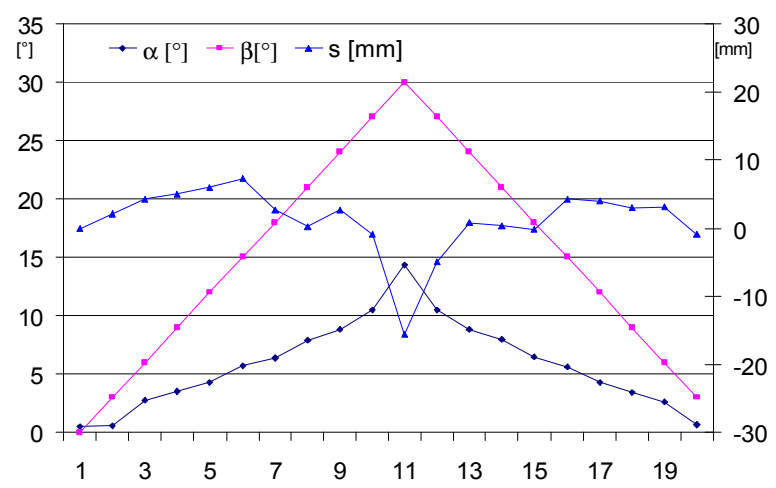

Fig. 10: Experimental set-up as in Fig. 9, but for negative rotations $\left(R_{-}\right)$at contact points $c_{1}, c_{2}$.

In the second experiment, the movement of the DLO is $R-$ at $c_{1}$ and also $R-$ at $c_{2}$. At both contact points, the given amount of the rotation is $3^{\circ}$ for every step. After rotating $30^{\circ}(10 \mathrm{steps})$ the inverse motion (at $c_{1}: R+$ and at $c_{2}: R+$ ) is performed. Fig. 10 shows that the resulting motion is about half the size of the desired motion. This is because of the material of the DLO. The steel ruler is too stiff to be approximated as a typical Spline.

\section{MULTIPLE POINT CONTACTS}

To solve the inverse manipulation problem for multiple 
contact points (number $n>2$ ), we use the same concept as presented in the previous section. The effect point $e$ is located between two contact points $c_{\mathrm{i}-1}, c_{\mathrm{i}}, 2<i \leq n$ and the DLO has a small contact pressure at every contact point. The positions of all contact points $c_{\mathrm{i}}$ are known. In addition, we know at least two tangents $T_{\mathrm{j}}, T_{\mathrm{k}}$ of the DLO at two arbitrary contact points $c_{\mathrm{j}}, c_{\mathrm{k}}(1 \leq j \leq n, 1 \leq k \leq n, j$ $\neq k)$. The other tangents $T_{\mathrm{i}}(1 \leq i \leq n, j \neq i \neq k)$ can be derived from the condition that the tangents and curvatures of two neighbouring Splines are identical.

Between two neighbouring contact points $c_{\mathrm{i}-1}$ and $c_{\mathrm{i}}, 2$ $<i \leq n$, the shape of the DLO is approximated by a Spline (see Fig. 11). Since we know two conditions of every Spline $\left(c_{\mathrm{i}-1}\right.$ and $\left.c_{\mathrm{i}}\right)$, we use the two given tangents to calculate all Splines. If we have not enough conditions available for one Spline, we need to calculate the second derivative at the neighbouring contact points and use this as additional condition. This is done with an equation system. For every Spline, we have four unknown three dimensional variables (twelve unknown variables) and with the two given tangents twelve equations for every Spline. With that information, we build a $12 n \times 12 n-$ matrix $A$ and one $12 n$ dimensional vector $b$. The result of the equation system is stored in $12 n$ dimensional result vector $x$.

$$
A^{n \times n} x^{n}=b^{n}
$$

This equation system can be solved with a Gauss algorithm. From this, we receive the vector $x$ with the necessary information about the Splines.

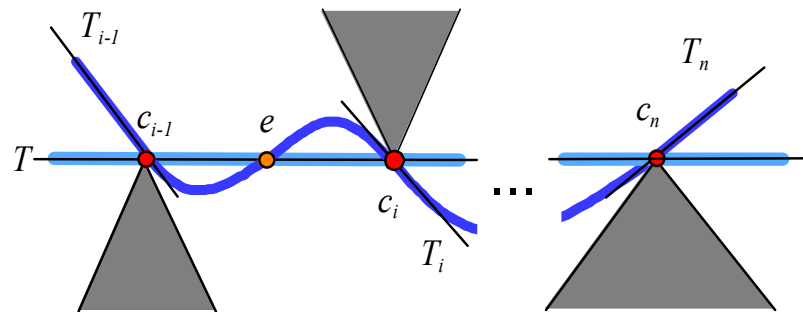

Fig. 11: DLO with multiple contact points $c_{\mathrm{i}}$, corresponding tangents $T_{\mathrm{i}}(1 \leq i \leq n)$, and effect point $e$.

The motion of the robot can be calculated from the Spline using the motion of the effect point at the Spline. This is similar to the motion calculation at two contact points and is therefore not further explained in this section.

To calculate the loss of a contact point, the normal vector $N_{i}=T_{i} \times N_{i}$ of every contact point $c_{\mathrm{i}}$ is used to detect whether contact is lost at $c_{\mathrm{i}}$ during the given motion (see Section 3 ). The movement of the effect point $e$ for rotation and translation is performed in the same way as in the previous section.

In the experiment, we use the set-up shown in Fig. 13. Please note that the DLO is fixed to $c_{3}$ so it could not lose that contact point no matter which movement is done in $e$. The movement is specified by the tangents $T_{1}$ and $T_{3}$ at $c_{1}$ and $c_{3}$. In Fig. 12, the measured rotations of the tangents $T_{i}\left(\alpha, \beta\right.$ and $\gamma$ for $c_{1}, c_{2}$ and $\left.c_{3}\right)$ and the calculated rotations (by S-Move) of the tangents $T_{i}{ }^{\prime}$ are shown $\left(\alpha^{\prime}, \beta^{\prime}\right.$ and $\gamma^{\prime}$ for $c_{1}, c_{2}$ and $c_{3}$ ). The amount of rotation at $c_{1}(\alpha)$ and $c_{3}$ $(\gamma)$ is about half of the desired value (like Fig. 10 with two contact points). The amount of rotation at $c_{2}(\beta)$ is shifted for the constant amount of about $20^{\circ}$ compared to the desired rotation. This shift is because of the used standard Spline. Other kinds of Splines are able to calculate that amount more precisely.

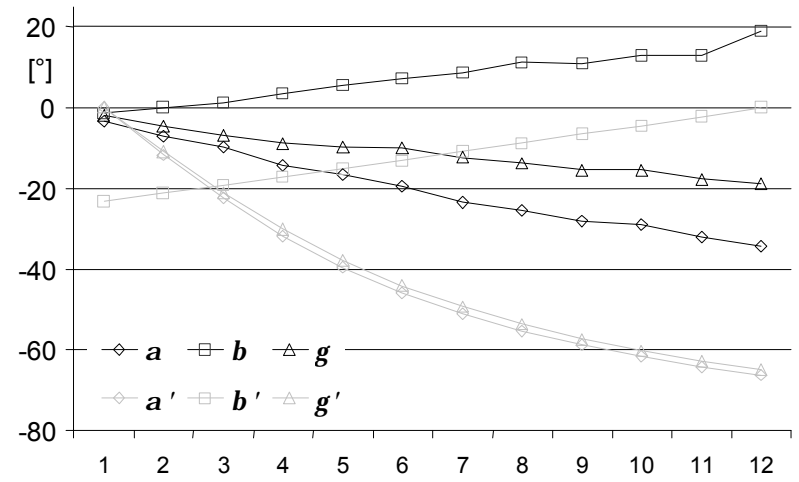

Fig. 12: Experimental results for three contact points and given rotations of $\alpha, \gamma$ at $c_{1}, c_{3}$. The angles $\alpha, \beta$ and $\gamma$ (for $c_{1}$, $c_{2}$ and $c_{3}$ ) show the measured rotation, and $\alpha^{\prime}, \beta^{\prime}$ and $\gamma^{\prime}$ indicates the calculated rotations by S-Move.

It is possible to specify the amount of DLO motion at every contact point. But the more tangents are given the more difficult it will be to deform the DLO in the desired way. Because, with every additional specified tangent there are three more equations. As a result, there are more equations than unknown variables. This equation system is still solvable, but it describes a DLO needing more than one effect point to change its shape in the specified manner.

A Spline calculated by S-Move and a real DLO are shown in Fig. 13. The figure shows the results of the experiment with a direct shape comparison. The calculated shape is only a rough approximation of the real DLO. Thus, the usage of weighted Splines or minimal energy Splines should be considered if a higher accuracy is needed to perform the given tasks.

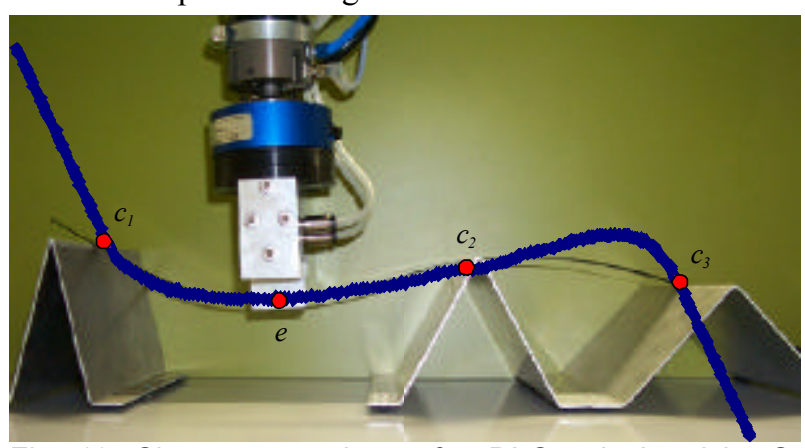

Fig. 13: Shape comparison of a DLO calculated by $\mathrm{S}$ Move $\left(T_{1}=(0,100,-100), T_{2}=(0,100,-1)\right.$, thick line $)$ with a real DLO. 
For multiple contact points, also weighted Splines, Splines under tension, and normalized Splines under tension are investigated in the same way as for standard Splines. These Splines are more accurate and more stable regarding degradation. Furthermore, the computing time is the smaller the more accurate these Splines are, but the mathematical formulations become more complex too. For details see [Schmidt01] and for a comparison see Fig. 14.

\begin{tabular}{|c|c|c|c|c|}
\hline & $\begin{array}{l}\text { standard- } \\
\text { Splines }\end{array}$ & $\begin{array}{l}\text { weighted } \\
\text { Splines }\end{array}$ & $\begin{array}{l}\text { Splines } \\
\text { under } \\
\text { tension }\end{array}$ & $\begin{array}{c}\text { normalized } \\
\text { Splines } \\
\text { under } \\
\text { tension } \\
\end{array}$ \\
\hline $\begin{array}{c}\text { number of } \\
\text { tangents }\end{array}$ & 2 & 2 & 2 & $m=0, \ldots, n$ \\
\hline $\begin{array}{l}\text { specified tan- } \\
\text { gent position }\end{array}$ & any desired & $\begin{array}{c}\text { near effect } \\
\text { point }\end{array}$ & $\begin{array}{c}\text { near effect } \\
\text { point }\end{array}$ & any desired \\
\hline $\begin{array}{l}\text { number of } \\
\text { parameters }\end{array}$ & $n+2$ & $2 n+1$ & $2 n$ & $3 n-3+m$ \\
\hline $\begin{array}{l}\text { effect point } \\
\text { position }\end{array}$ & $\begin{array}{l}\text { not re- } \\
\text { garded }\end{array}$ & $\begin{array}{l}\text { influences } \\
\text { damping }\end{array}$ & $\begin{array}{c}\text { influences } \\
\text { damping }\end{array}$ & $\begin{array}{c}\text { influences } \\
\text { damping }\end{array}$ \\
\hline $\begin{array}{l}\text { optimal num- } \\
\text { ber of contact } \\
\text { points }\end{array}$ & 2 & $2, \ldots, 4$ & $2, \ldots, 12^{\#}$ & $2, \ldots, 26^{\#}$ \\
\hline degradation & yes & yes & sometimes & rare \\
\hline damping & none & conditional & yes & yes \\
\hline $\begin{array}{c}\text { material } \\
\text { parameter }\end{array}$ & none & none & yes & yes \\
\hline complexity & $3312(n-1)^{2}$ & $3312(n-1)^{2}$ & $72 \cdot n^{2}$ & $45 \cdot(n+2)^{2}$ \\
\hline
\end{tabular}

Fig. 14: Comparison of Splines with different number of parameters for $n$ contact points ( ${ }^{*}$ partly empirical; ${ }^{*}$ limited by the resources of the used robot) [Schmidt01]

\section{CONCLUSIONS}

For single point contacts, CP-Move solves the direct and inverse manipulation problem only heuristically. An analytical approach for the more important inverse problem is provided by RT-Move. Additionally, RT-Move enables the user to specify not only qualitatively but also quantitatively the desired DLO motion in the contact points. Furthermore, RT-Move is able to reverse the movements and deformations of the DLO.

For multiple points contacts, S-Move provides a very general and analytical solution for the inverse problem. The desired DLO motion S-Move can be specified quantitatively. Additionally, we are able to calculate whether a contact is lost at one of the contact points. Finally, SMove can be used to solve the direct manipulation problem too [Schmidt01]. With this, S-Move provides a good basis for assembly tasks with wires or hoses were every contact of a DLO with an obstacle is regarded as a contact point.

There are three further questions that are interesting to be examined. First, are there other functions with a better physical DLO approximation than Splines (e.g. weighted Spline interpolation or minimal energy Splines)? Second, how can DLOs with a curved shape be approximated correctly. Third, will the use (force or vision) sensors improve the results of the presented algorithms for multiple point contacts?

\section{REFERECES}

[Henrich00a] Henrich D., Wörn H. (Eds.): "Robot manipulation of deformable objects", Advanced Manufacturing Series, Springer-Verlag, London, 2000, ISBN: 185233-250-6.

[Henrich99a] Henrich D., Ogasawara T., Wörn H.: "Manipulating deformable linear objects - Contact states and point contacts". In: 1999 IEEE International Symposium on Assembly and Task Planning (ISATP'99), Porto, Portugal, July 21 - 24, 1999, pp 198-204

[Kraus97] Kraus W., McCarragher B.J.: "Force fields in the manipulation of flexible materials", In: Proc. IEEE Int. Conf. on Robotics and Automation (ICRA'96), vol. 3, pp. 2352-2357, Minneapolis, April 1996.

[Nakagaki95]Nakagaki H., Kitagaki K., Tsukune H.: "Study of insertion task of a flexible beam into a hole". In: Proc. of IEEE Int. Conf. on Robotics and Automation (ICRA'95), vol. 1, pp. 330-3351, 1995.

[Nakagaki96]Nakagaki H., Kitagaki K., Ogasawara T., Tsukune H.: "Study of insertion task of a flexible beam into a hole by using visual tracking observed by stereo vision". In: Proc. of IEEE Int. Conf. on Robotics and Automation (ICRA'96), vol. 4, pp. 3209-3214, 1996.

[Remde99c] Remde A., Henrich D., Karl S., Wörn H.: "Manipulating deformable linear objects: Efficient simulation of the workpiece behavior". In: Proc. IASTED Int. Conf. Robotics and Applications (RA'99), pp 64-70, Oct. 28-30, 1999 Santa Barbara, IASTED/Acta Press

[Schmidt01] Schmidt, T.: "Manipulation deformierbarer linearer Objekte - Roboterbewegungen in Einzel- und Mehrpunktkontakten" ("Manipulating Deformable Linear Objects Robot Motions in Single and Multiple Contact Points"), Diploma Thesis, Informatics Faculty, University Kaiserslautern, 2001.

[Suehiro89] Suehiro T., Takase K.: "Representation and control of motion in contact and its application to assembly tasks". In: Proc. 5th Int. Symp. on Robotics Research, 1989.

[Wakamatsu95] Wakamatsu H., Hirai S., Iwata K., "Modeling of linear objects considering bend, twist, and extensional deformations". In: Proc. IEEE Int. Conf. on Robotics and Automation (ICRA'95), vol. 1, pp. 433438, Nagoya, May, 1995.

[Wakamatsu97] Wakamatsu H., Matsumura T., Arai E., Hirai S.: "Dynamic Analysis of string objects deformation for their manipulation". In: Proc. IEEE/RSJ Int. Conf. on Intelligent Robots and Systems (IROS'97), pp. 196-201, Grenoble, Sept., 1997.

[Zheng91] Zheng Y. F., Pei R., Chen C.: "Strategies for automation assembly of deformable objects". In: IEEE Int. Conf. on Robotics and Automation, 1991, pp. 2598- 2603. 